OPEN ACCESS

Edited by:

Shuo Wang,

Capital Medical University, China

Reviewed by:

Steffen Tiedt, LMU Munich University Hospital,

Germany

Jaime Ramos-Cejudo

New York University, United States

${ }^{*}$ Correspondence:

Qingsong Wang wqscdgh@sina.com

Dongdong Yang

1241668186@qq.com

Received: 20 November 2020 Accepted: 22 January 2021

Published: 19 February 2021

Citation:

Wang Z, Wang R, Li Y, Li M, Zhang Y, Jiang L, Fan J, Wang $Q$ and Yang D (2021) Plasma Neurofilament Light Chain as a Predictive Biomarker for Post-stroke Cognitive Impairment:

A Prospective Cohort Study.

Front. Aging Neurosci. 13:631738.

doi: 10.3389/fnagi.2021.631738

\section{Plasma Neurofilament Light Chain as a Predictive Biomarker for Post-stroke Cognitive Impairment: A Prospective Cohort Study}

\author{
Zhiqiang Wang ${ }^{1,2}$, Rongyu Wang ${ }^{1,2}$, Yuxia Li ${ }^{3}$, Mao $\mathrm{Li}^{1,2}$, Yaodan Zhang ${ }^{1,2}$, \\ Lianyan Jiang ${ }^{1,2}$, Jin Fan' ${ }^{2}$, Qingsong Wang ${ }^{3 *}$ and Dongdong Yang ${ }^{1 *}$ \\ ${ }^{1}$ Department of Neurology, Hospital of Chengdu University of Traditional Chinese Medicine, Chengdu, China, ${ }^{2}$ School \\ of Clinical Medicine, Chengdu University of TCM, Chengdu, China, ${ }^{3}$ Department of Neurology, The General Hospital \\ of Western Theater Command, Chengdu, China
}

Background: Plasma neurofilaments light chain (pNfL) is a marker of axonal injury. The purpose of this study was to examine the role of pNfL as a predictive biomarker for post-stroke cognitive impairment (PSCl).

Methods: A prospective single-center observational cohort study was conducted the General Hospital of Western Theater Command between July 1, 2017 and December 31,2019 . Consecutive patients $\geq 18$ years with first-ever acute ischemic stroke (AIS) of anterior circulation within $24 \mathrm{~h}$ of symptom onset were included. PSCl was defined by the Montreal Cognitive Assessment (MOCA) (MOCA < 26) at 90 days after stroke onset.

Results: A total of 1,694 patients [male, 893 (52.70\%); median age, 64 (16) years] were enrolled in the cohort analysis, and 1,029 (60.70\%) were diagnosed with PSCl. Patients with PSCI had significantly higher pNfL [median (IQR), 55.96 (36.13) vs. 35.73 (17.57) $\mathrm{pg} / \mathrm{ml} ; P<0.001]$ than Non-PSCl. pNfL was valuable for the prediction of PSCl (OR 1.044, 95\% Cl 1.038-1.049, $P<0.001)$ after a logistic regression analysis, even after adjusting for conventional risk factors including age, sex, education level, NIHSS, TOAST classification, and infarction volume (OR 1.041, 95\% Cl 1.034-1.047, $P<0.001$ ). The optimal cutoff value of the $\mathrm{pNfL}$ concentration was $46.12 \mathrm{pg} / \mathrm{ml}$, which yielded a sensitivity of $71.0 \%$ and a specificity of $81.5 \%$, with the area under the curve (AUC) at $0.785(95 \% \mathrm{Cl} 0.762-0.808, P<0.001)$.

Conclusion: This prospective cohort study showed that the pNfL concentration within $48 \mathrm{~h}$ of onset was an independent risk factor for $\mathrm{PSCl} 90$ days after an anterior circulation stroke, even after being adjusted for potential influencing factors regarded as clinically relevant.

Clinical Trial Registration: www.chictr.org.cn, identifier ChiCTR1800020330. 


\section{INTRODUCTION}

Stroke survivors are at increased risk for cognitive impairment. Studies have shown that even mild strokes can increase the risk of cognitive impairment in survivors and affect their quality of life (Fride et al., 2015). Epidemiological studies have shown that stroke causes cognitive dysfunction in approximately one-third of patients, but it is unknown which stroke patients will suffer from cognitive impairment. Thus, the diagnosis and prediction of post-stroke cognitive impairment (PSCI) functional recovery by biomarkers has become a hot research topic. Previous studies have indicated that inflammatory biomarkers, growth factors, oxidative damage biomarkers, genetic biomarkers, and metabolic biomarkers in the circulating blood of patients may be the key determinants for the diagnosis and prediction of cognitive impairment (Zhang and $\mathrm{Bi}, 2020$ ). However, these markers cannot reflect the mechanism of cognitive impairment caused by a stroke. The direct pathological cause of PSCI is neuronal damage in key brain regions, so looking for markers related to neuronal damage may predict and reflect a decline in the cognitive level.

Neurofilament light chain (NfL) is a neuron-specific structural protein (Zetterberg, 2016) that has recently been suggested as a marker of axonal injury and neurodegeneration with potential applications for both patient monitoring and for observational and interventional studies (Tiedt et al., 2018). In recent years, with the development of the quantitative detection technology of plasma NfL ( $\mathrm{pNfL}$ ), studies regarding the role of $\mathrm{pNfL}$ in neurodegenerative diseases and brain injury have been increasing (Gattringer et al., 2017; Guedes et al., 2020; Quiroz et al., 2020). Recent studies have observed a significant correlation among pNfL and the National Institutes of Health Stroke Scale (NIHSS) upon admission (Traenka et al., 2015; Al-Khaled, 2018), age-related white matter changes, infarct volume (Gattringer et al., 2017; Tiedt et al., 2018; Onatsu et al., 2019) and clinical outcome 90 days after stroke (Uphaus et al., 2019). A metaanalysis showed that the pNfL was a promising predictive biomarker for ischemic stroke outcome (Liu et al., 2020). The pNfL concentration had also been considered to be related to cognitive deterioration (Zetterberg et al., 2016; Mattsson et al., 2017; Olsson et al., 2019). To date and to our knowledge, the usefulness of pNfL for understanding PSCI is unclear. To address this, a prospective single-center observational cohort study was conducted to determine the association between pNfL and PSCI.

\section{MATERIALS AND METHODS}

\section{Study Design and Participants}

This was a prospective single-center observational cohort study that included consecutive patients $\geq 18$ years with first ever AIS of the anterior circulation within $24 \mathrm{~h}$ of symptom onset who were admitted to the General Hospital of Western Theater Command between July 1, 2017 and December 31, 2019. AIS was diagnosed according to the World Health Organization criteria and confirmed using brain computed tomography (CT) or magnetic resonance imaging (MRI). Patients were excluded if they (1) had a pre-existing cognitive impairment (clinical diagnosis or previous treatment or if the subject/caregiver reported progressive forgetfulness), mental illness or were unable to complete the cognitive assessments; (2) had issues combined with other non-vascular causes of neural function defects (brain injury, Alzheimer's disease, Parkinson's disease, and other neurological diseases);(3) were accompanied by serious medical diseases, tumor, hepatitis or an autoimmune disease; or (4) had survived less than 3 months.

Stroke severity was assessed using the National Institutes of Health Stroke Scale Score (NIHSS) and infarct volume (calculated using the MRI-DWI). The DWI lesion volumes were determined by the consensus of two experienced raters unaware of the clinical and laboratory results. The lesion size was calculated using the commonly used semiquantitative method (Broderick et al., 1993). All of the cases were invited for a 90-day follow-up visit. A physician blinded to the clinical data assessed changes in cognition. Post-stroke cognitive impairment (PSCI) was defined by the Montreal Cognitive Assessment (MOCA) (MOCA < 26) at 90 ( \pm 5 ) days (Lees et al., 2014) after stroke onset via in-person interview.

\section{Blood Sampling and Biomarker Measurements}

Venous blood samples were drawn upon admission or the next morning (within $48 \mathrm{~h}$ of stroke onset), and the time from stroke onset to blood collected was recorded. After centrifugation for $20 \mathrm{~min}$ at 3,000 $\mathrm{g}$ at room temperature, plasma (from the ethylene diamino tetraacetic acid (EDTA) tube) was aliquoted. The tubes were frozen locally at $-80^{\circ} \mathrm{C}$ within 40 min after collection. The pNfL was measured using a single-molecule assay (SiMoA) platform (Quanterix, Lexington, MA, United States) as described (Weston et al., 2017; Tiedt et al., 2018). An in-house pool was used as an internal control and included in each assay for evaluating the assay performance. Samples were analyzed in duplicates, and the coefficient of variation $(\mathrm{CV})$ was $<11 \%$.

\section{Statistical Analysis}

Discrete variables were expressed as counts (percentages) and continuous variables as medians (interquartile range [IQR]). For the univariate analysis, the Mann-Whitney $U$ test or chi-squared test was used to compare the demographic and clinical characteristics of patients with and without PSCI, as appropriate. The pNfL, MOCA, infarct volume, and NIHSS scores were log transformed (based 10) to closely normal distributions. In order to test for significant correlations between the clinical characteristics of patients and the plasma data, the Pearson correlational was used. The association of the pNfL levels upon admission with cognitive impairment was analyzed using a multiple logistic regression and adjusted for the established predictors. Variables that were identified as significant in univariate analyses $(P<0.1)$ were entered into the regression analyses together with other clinically significant variables. The optimal cutoff levels for the dichotomizing values were selected as the situation maximizing the Youden index. The receiver operating characteristic (ROC) analysis was performed 
TABLE 1 | Baseline characteristics of the participants.

\begin{tabular}{|c|c|c|c|c|}
\hline Factors & Total & PSCI & Non-PSCI & $P$ \\
\hline Sex, male, $n(\%)$ & 893 (52.72) & $538(52.28)$ & 355 (53.38) & 0.658 \\
\hline Education level, $<6$ years, $n$ (\%) & $900(53.13)$ & $561(64.52)$ & 339 (50.98) & 0.154 \\
\hline $\mathrm{BMI}\left(\mathrm{kg} / \mathrm{m}^{2}\right)$ & $24.09(1.53)$ & $24.22(1.59)$ & $23.92(1.43)$ & $<0.001$ \\
\hline \multicolumn{5}{|l|}{ Vascular risk factors, $n(\%)$} \\
\hline Diabetes mellitus & $566(33.41)$ & $346(33.62)$ & $220(33.08)$ & 0.817 \\
\hline Hyperlipidemia & 385 (22.73) & $249(24.20)$ & $136(20.45)$ & 0.072 \\
\hline Atrial fibrillation & $371(21.90)$ & 267 (25.95) & $104(15.64)$ & $<0.001$ \\
\hline Smoking & $478(28.22)$ & $276(26.82)$ & $202(30.38)$ & 0.113 \\
\hline Drinking & $318(18.77)$ & $194(18.85)$ & $124(18.65)$ & 0.915 \\
\hline NIHSS, median (IQR) & $4(7)$ & $6(8)$ & $3(3)$ & $<0.001$ \\
\hline Cardioembolism & $239(14.11)$ & $175(17.01)$ & $64(9.62)$ & \\
\hline Small vessel occlusion & $154(9.09)$ & $83(8.07)$ & $71(10.68)$ & \\
\hline Other cause & $68(4.01)$ & $45(4.37)$ & $23(3.46)$ & \\
\hline Undetermined & 305 (18.01) & $178(17.30)$ & $127(19.10)$ & \\
\hline Blood sampling time (h), median (IQR) & $19.00(19.00)$ & $20.00(19.25)$ & $17.00(19.00)$ & 0.471 \\
\hline pNfL (pg/mL), median (IQR) & $46.41(36.26)$ & $55.96(36.13)$ & $35.73(17.57)$ & $<0.001$ \\
\hline HbA1c (\%), median (IQR) & $5.9(1.10)$ & $5.9(1.20)$ & $5.9(0.80)$ & 0.901 \\
\hline HsCRP (mg/L), median (IQR) & $3.58(2.75)$ & $3.51(2.71)$ & $3.61(2.87)$ & 0.332 \\
\hline $\mathrm{HCY}(\mu \mathrm{mol} / \mathrm{L})$, median (IQR) & $14.64(9.45)$ & $15.18(9.24)$ & $14.03(10.15)$ & $<0.001$ \\
\hline MOCA, median (IQR) & $24(7)$ & $21(8)$ & $27(2)$ & $<0.001$ \\
\hline
\end{tabular}

PSCI, post-stroke cognitive impairment; IQR, interquartile range; BMI, Body Mass Index; pNfL, plasma neurofilament light chain concentration; NIHSS, National Institutes of Health Stroke Scale; and TOAST, Trial of ORG 10172 in Acute Stroke Treatment; HsCRP, high sensitivity C-reactive protein; HCY, homocysteine; MOCA, Montreal Cognitive Assessment. Bold text indicates a statistically less than 0.05.

to determine the sensitivity, specificity, and area-under-the ROC curve of the pNfL for detection of PSCI. In order to validate the model, 5-fold cross-validation was employed using the RMS package available on $\mathrm{R}$ statistical software. Other analyses were performed using SPSS 22 (IBM, Chicago, IL). All of the tests were 2 -sided, and a $P<0.05$ was considered to be significant.

\section{RESULTS}

A comparison of the demographic and clinical variables in the final dataset, including the pNfL data, is shown in Table 1. Of the 1,896 patients who were initially screened for eligibility, 202 (10.65\%) were excluded for reasons that included: $68(3.59 \%)$ death within 3 months, $45(2.37 \%)$ combined with other central nervous system diseases, $43(2.27 \%)$ unable to complete the cognitive assessments, $12(0.63 \%)$ no plasma available, and 34 (1.79\%) withdrew consent or were lost to follow-up. Finally, a total of 1,694 patients [male, 893 (52.72\%); median age, 64 (IQR, 16) years] were enrolled in the cohort analysis, the median MOCA was 24 (IQR, 7), the median NIHSS was 4 (IQR, 7), the median blood sampling time was $19 \mathrm{~h}$ (IQR, 19; range 2-48), and the median pNfL was 46.41 (IQR, 36.26) pg/ml. A total of 1,029 (60.74\%) were diagnosed with PSCI.

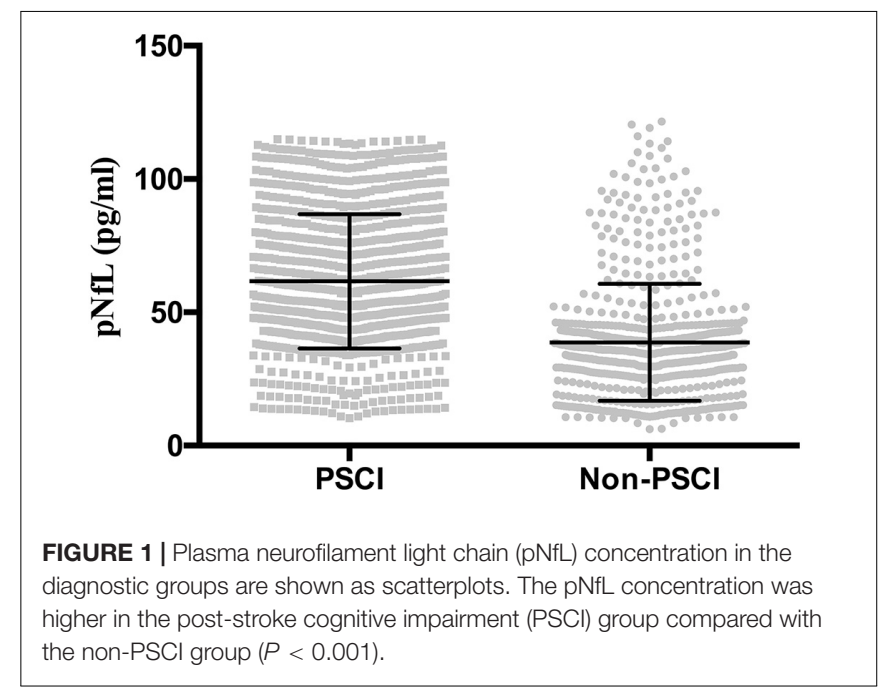

The PSCI and non-PSCI patients were well matched for sex, education level, history of hypertension, hyperlipidemia, diabetes, smoking, alcohol (all $P>0.05$ ). However, the participants with atrial fibrillation, of older age, more clinical severity on the NIHSS, a larger infarction volume (ml), or a 
higher body mass index (BMI) were more common in the PSCI group $(P<0.05)$. No significant difference was observed between the two groups in terms of time to blood sampling (h), plasma high sensitivity C-reactive protein (HsCRP) level, and HbA1c levels (all $P>0.05$ ). Compared with the non-PSCI group, the PSCI group exhibited significantly higher levels of pNFL and homocysteine (HCY) (both $P<0.05)$ (Table 1 and Figure 1 ).

Correlation analysis showed that the $\log _{10}$ pNfL levels correlated with age $(r=0.130, P<0.001), \log _{10}$ cerebral infarction volumes $(r=0.509, P<0.001$; Figure $2 \mathrm{~A})$, the $\log _{10}$ NIHSS score $(r=0.510, P<0.001$; Figure $2 B)$, and the $\log _{10}$ time to blood sampling ( $r=0.261, P<0.001$; Figure 2C). The $\log _{10}$ pNfL levels were negatively correlated with cognitive impairment defined by $\log _{10} \operatorname{MOCA}(r=-0.523, P<0.001$; Figure 2D).

A multivariable logistic regression revealed that patients with higher pNfL had a significantly higher risk of PSCI, even after adjusting for conventional risk factors including age, sex, education level, NIHSS score, TOAST classification and infarction volume $(P<0.05)$. This result indicated that the $\mathrm{pNfL}$ could be an independent risk factor for PSCI (Table 2).

The optimal cutoff value of the pNfL concentration as an indicator for auxiliary diagnosis of PSCI was assessed using the ROC curve. The optimal threshold was $46.12 \mathrm{pg} / \mathrm{ml}$, which yielded a sensitivity of $71.0 \%$ and a specificity of $81.5 \%$, with the AUC at 0.785 (95\% CI, 0.762-0.808; $P<0.001$; Figure 3). After 5 -fold cross-validation, the $\mathrm{c}$-statistic of the model was still 0.785 .
The calibration curve was very close to the actual curve, which showed that the model fits well.

\section{DISCUSSION}

This prospective cohort study showed that the pNfL concentration within $48 \mathrm{~h}$ of onset was an independent risk factor for PSCI within 90 days after an anterior circulation stroke, even after adjustment for potential influencing factors regarded as clinically relevant. In the present study, it was found that PSCI patients exhibited higher pNfL and Hcy levels than non-PSCI patients. Ages, BMI, atrial fibrillation, clinical severity on the NIHSS, and the infarction volume were also associated with cognitive function in post-stroke patients. The pNfL was positively correlated with the NIHSS score, infarct volume and the time to blood sampling time, negatively correlated with the MOCA. The levels of pNfL showed significant diagnostic accuracy in discriminating patients with PSCI from those without PSCI. This is the first study that has investigated the pNfL levels in patients with PSCI.

NfL, a neuron-specific structural protein (Zetterberg, 2016), has recently been suggested as a marker of neuroaxonal injury after ischemic stroke with potential application prospects for patient monitoring, observation, and intervention studies (Tiedt et al., 2018). Cerebrospinal fluid (CSF) NfL concentrations can
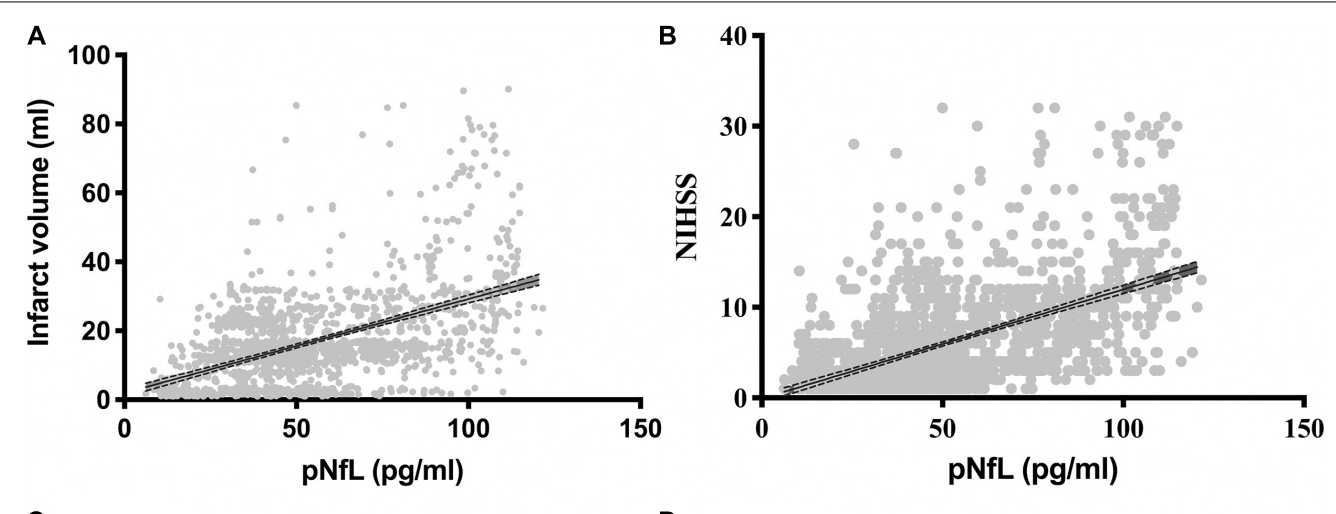

C

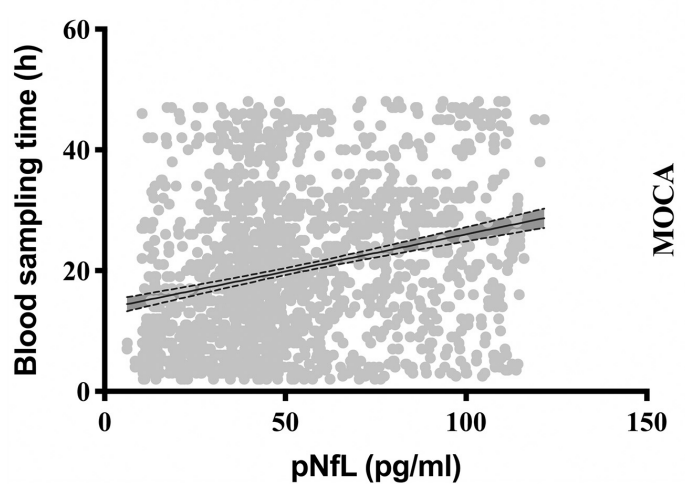

D

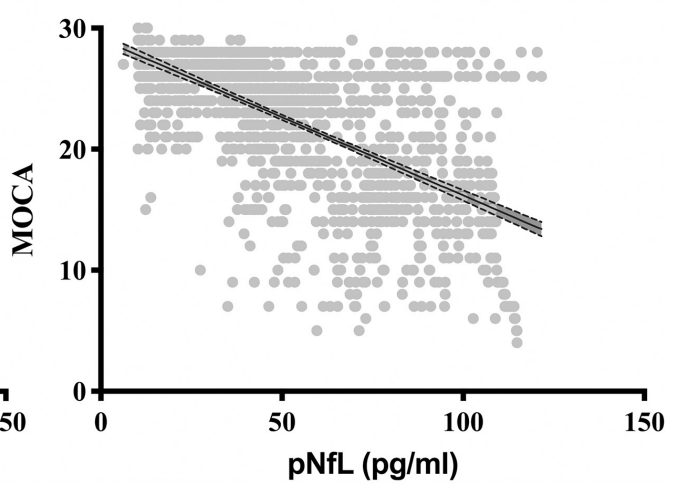

FIGURE 2 | Correlations of the plasma neurofilament light chain (pNfL) concentration with (A) the infarction volume (ml), (B) the National Institutes of Health Stroke Scale (NIHSS), (C) the blood sampling time (h), and (D) the Montreal Cognitive Assessment (MOCA). 
TABLE 2 | Logistic regression analysis for the association of pNfL with PSCl at 90-days.

\begin{tabular}{|c|c|c|c|}
\hline Variables & OR & $95 \% \mathrm{Cl}$ & $P$ \\
\hline Unadjusted pNfL & 1.044 & $1.038-1.049$ & $<0.001$ \\
\hline Model 1 pNfL & 1.044 & $1.038-1.050$ & $<0.001$ \\
\hline Model 2 pNfL & 1.041 & $1.034-1.047$ & $<0.001$ \\
\hline
\end{tabular}

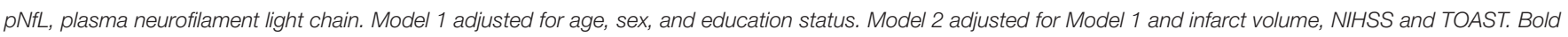
text indicates a statistical significance of less than 0.05 .

be used as markers of axonal damage in white matter and other subcortical brain structures (Lycke et al., 1998; Zetterberg et al., 2006). Previous studies have shown that NfL expression was associated with dementia (Rosengren et al., 1999; de Jong et al., 2007; Howell et al., 2017; Zhao et al., 2019), small vessel disease (Gattringer et al., 2017), and other neurodegenerative diseases (Ge et al., 2018; Khalil et al., 2018; Bridel et al., 2019; Gagliardi et al., 2019; Gao et al., 2020). In addition, an increasing number of studies have demonstrated that pNfL levels were associated with clinical characteristics and outcome in stroke patients (Tiedt et al., 2018; Uphaus et al., 2019; Nielsen et al., 2020), and the CSF NfL increased months before the first dementia symptoms appeared, suggesting it might serve as a preclinical marker (Bacioglu et al., 2016). However, whether NfL expression is related to the occurrence of PSCI is still unknown. This study is the first to show that pNfL concentration was a blood marker of PSCI and has significant diagnostic accuracy in discriminating patients with PSCI from those without PSCI. The exclusion criteria of this study were not harsh; for example, patients with cortical infarction, large infarct size, or specific causes of stroke were not excluded. Hence, these findings could be considered representative of the spectrum of PSCI.

This study was the first to reveal the correlation between the NfL expression in plasma and the occurrence of PSCI. Previous studies have shown that the expression of NfL in the cerebral spinal fluid (CSF) was correlated with cognitive function, including Alzheimer's disease (AD) (Weston et al., 2017), and frontotemporal dementia (FTD) (Rohrer et al., 2016), even in a small sample of vascular dementia (VaD) studies (Rosengren et al., 1999; Skillbäck et al., 2014). A network meta-analysis further demonstrated a significant increase in the CSF NfL expression level in dementias that engage the subcortical brain regions, such as $\mathrm{VaD}$, than other types of dementia (Skillbäck et al., 2014; Zhao et al., 2019). Because the CSF collection is relatively complex, especially for stroke patients, the development of blood biomarkers (such as exosomes) is particularly important, and it is an important target for the future study of PSCI markers. The recent development of methods to quantify NfL in plasma had demonstrated that the pNfL concentration was closely correlated with cerebrospinal fluid NfL and directly reflected neurodegeneration within the central nervous system (Palermo et al., 2020). Consistent with what would be expected of a marker of neuronal damage, results from these studies showed a higher pNfL in patients with PSCI and correlations between pNfL and PSCI were observed (Gattringer et al., 2017; Tiedt et al., 2018). In agreement with earlier studies, it was found in this study that the severity of cognitive impairment increased with increasing pNfL levels (Gendron et al., 2020),

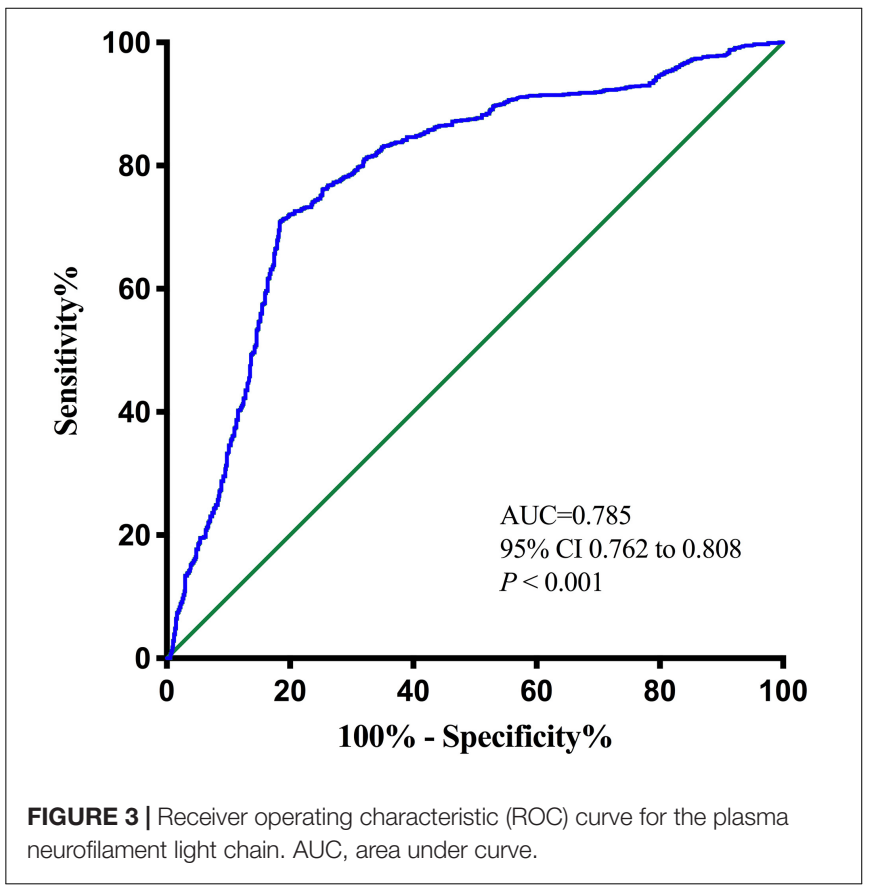

making pNfL an easily accessible biomarker of the progression of neurodegenerative dementia diseases (Mattsson et al., 2017; Weston et al., 2017; Olsson et al., 2019).

In this study, a prospective cohort study was designed to show that pNfL was an independent risk factor for PSCI. Previous small-sample case-control studies only observed increased NfL expression in CSF of VaD patients (Sjögren et al., 2001; Wallin and Sjögren, 2001; Skillbäck et al., 2014; Zhao et al., 2019). In this study, by using a prospective cohort design, the regression analysis showed that pNfL expression was an independent risk factor for PSCI. The risks of PSCI were associated with age and vascular risk factors, such as atrial fibrillation, which was consistent with a previous study (Pendlebury et al., 2019). Also, stroke severity and infarction volume should be considered (Tiedt et al., 2018). In this study, all parameters were included when assessing the correlation of PSCI and pNfL. Nevertheless, after controlling for the confounders, the pNfL level displayed as an independent predictor of PSCI. The role of NfL in the pathophysiology of PSCI might be through some possible signaling pathways. pNfL is related to stroke severity (NIHSS score and lesion volume) and clinical outcomes (Onatsu et al., 2019; Pedersen et al., 2019), which are independent predictors of PSCI. However, pNfL still was a predictor for PSCI after adjusting for the NIHSS score and lesion volume, which was consistent 
with the results of a recent study (Gendron et al., 2020). The potential reasons behind why $\mathrm{pNfL}$ adds an additional predictive value apart from stroke severity should be considered. Acute infarcts further induce secondary neurodegeneration outside the infarct area, such as white matter tracts connected to the infarct. That secondary damage could contribute to poor cognitive and blocked neurotransmitter synthesis that leads to PSCI.

This study identified the correlation between $\mathrm{pNfL}$ and PSCI within $48 \mathrm{~h}$ of onset. There is evidence that pNfL increases with the time from symptom onset to blood-draw (Traenka et al., 2015; Gattringer et al., 2017; Tiedt et al., 2018), illustrating that the time point of measurement is of great importance when evaluating pNfL (Al-Khaled, 2018; Tiedt et al., 2018). To control the influence of blood collection time on the results, blood was collected within $48 \mathrm{~h}$ after the onset of the disease. It should be noted that patients within $24 \mathrm{~h}$ after stroke symptoms onset were included in this study. However, as some patients had their blood collected the next morning, the overall blood collection time was $48 \mathrm{~h}$. This study showed that there was weak correlation between the concentration of $\mathrm{pNfL}$ and the blood collection time, and there was no difference in the blood collection time between groups. In contrast to a previous study that showed an association among functional outcomes 90 days after ischemic stroke and pNfL measured 7 days after symptom onset (Al-Khaled, 2018; Tiedt et al., 2018), as well as NfL that was independently correlated with the Mini-Mental State Examination at 0-8 days (Gendron et al., 2020), it was possible to already show a predictive effect of the NfL measured in plasma collected within $48 \mathrm{~h}$ after symptom onset. This was consistent with recent studies (Pedersen et al., 2019; Uphaus et al., 2019). This is especially important, as biomarker-based decision-making might be mandatory before the 7-day time point (Uphaus et al., 2019).

This study had several limitations. First, single-center cohorts, the exclusion of patients with aphasia or other severe conditions and patients in whom measurement of the pNfL levels failed may have led to an underestimation of the actual incidence of PSCI. Second, pNfL was measured only once. It may be essential to conduct a longitudinal study that measures at multiple time points after stroke to provide better prognostic information. Third, the centrifugal operation process was slightly different from the current standard guidelines (Tiedt et al., 2018), and the results should be further validated in future studies. Finally, single biomarkers may not be sufficient, and multiple biomarkers combined with a machine-learning algorithm should be used to automatically diagnose and predict PSCI.

\section{REFERENCES}

Al-Khaled, M. (2018). Serum neurofilament light as diagnostic biomarker for acute cerebral ischemia: a promising tool. Eur. J. Neurol. 25:609. doi: 10.1111/ene. 13588

Bacioglu, M., Maia, L. F., Preische, O., Schelle, J., Apel, A., Kaeser, S. A., et al. (2016). Neurofilament light chain in blood and CSF as marker of disease progression in mouse models and in Neurodegenerative Diseases. Neuron 91, 56-66. doi: 10.1016/j.neuron.2016.05.018

\section{CONCLUSION}

In conclusion, in this study, it was demonstrated that high pNfL levels within $48 \mathrm{~h}$ after first-ever anterior circulation stroke were associated with the development of PSCI 90 days after an acute ischemic stroke (AIS). In addition, this study showed significant diagnostic accuracy for discriminating patients with PSCI from patients without cognitive impairment. Further studies are needed to verify this association.

\section{DATA AVAILABILITY STATEMENT}

The raw data supporting the conclusions of this article will be made available by the authors, without undue reservation.

\section{ETHICS STATEMENT}

The studies involving human participants were reviewed and approved by the Ethics Committee of General Hospital of Western Theater Command. The patients/participants provided their written informed consent to participate in this study.

\section{AUTHOR CONTRIBUTIONS}

DY: conceptualization, methodology, and software. QW: conceptualization, methodology, supervision, and investigation. ZW: data curation, writing the original draft, reviewing, and editing. RW: data curation and writing the original draft. ML and LJ: data curation. YL: data curation and investigation. YZ: software and validation. JF: supervision. All the authors reviewed the manuscript and approved the submitted version.

\section{FUNDING}

This study was supported by the Scientific Research Project of Health and Family Planning Commission of Sichuan Province (No. 16PJ014).

\section{ACKNOWLEDGMENTS}

We thank all of the patients and their families for their participation in the study and the investigators.

Bridel, C., Van Wieringen, W. N., Zetterberg, H., Tijms, B. M., Teunissen, C. E., and Alvarez-Cermeño, J. C. (2019). Diagnostic value of cerebrospinal fluid neurofilament light protein in neurology: a systematic review and metaanalysis. JAMA Neurol. 76, 1035-1048.

Broderick, J. P., Brott, T. G., Duldner, J. E., Tomsick, T., and Huster, G. (1993). Volume of intracerebral hemorrhage. A powerful and easy-to-use predictor of 30-day mortality. Stroke 24, 987-993. doi: 10.1161/01.str.24.7.987

de Jong, D., Jansen, R. W., Pijnenburg, Y. A., Van Geel, W. J., Borm, G. F., Kremer, H. P., et al. (2007). CSF neurofilament proteins in the differential diagnosis of 
dementia. J. Neurol. Neurosurg. Psychiatry 78, 936-938. doi: 10.1136/jnnp.2006. 107326

Fride, Y., Adamit, T., Maeir, A., Ben Assayag, E., Bornstein, N. M., Korczyn, A. D., et al. (2015). What are the correlates of cognition and participation to return to work after first ever mild stroke? Top. Stroke Rehabil. 22, 317-325. doi: 10.1179/1074935714z.0000000013

Gagliardi, D., Meneri, M., Saccomanno, D., Bresolin, N., Comi, G. P., and Corti, S. (2019). Diagnostic and prognostic role of blood and cerebrospinal fluid and blood neurofilaments in amyotrophic lateral sclerosis: a review of the literature. Int. J. Mol. Sci. 20:4152. doi: 10.3390/ijms20174152

Gao, W., Zhang, Z., Lv, X., Wu, Q., Yan, J., Mao, G., et al. (2020). Neurofilament light chain level in traumatic brain injury: a system review and meta-analysis. Medicine 99:e22363. doi: 10.1097/md.0000000000022363

Gattringer, T., Pinter, D., Enzinger, C., Seifert-Held, T., Kneihsl, M., Fandler, S., et al. (2017). Serum neurofilament light is sensitive to active cerebral small vessel disease. Neurology 89, 2108-2114. doi: 10.1212/wnl.0000000000004645

Ge, F., Ding, J., Liu, Y., Lin, H., and Chang, T. (2018). Cerebrospinal fluid NFL in the differential diagnosis of parkinsonian disorders: a meta-analysis. Neurosci. Lett. 685, 35-41. doi: 10.1016/j.neulet.2018.07.030

Gendron, T. F., Badi, M. K., Heckman, M. G., Jansen-West, K. R., Vilanilam, G. K., and Johnson, P. W. (2020). Plasma neurofilament light predicts mortality in patients with stroke. Sci. Transl. Med. 12:eaay1913.

Guedes, V. A., Kenney, K., Shahim, P., Qu, B. X., Lai, C., Devoto, C., et al. (2020). Exosomal neurofilament light: a prognostic biomarker for remote symptoms after mild traumatic brain injury? Neurology 94, e2412-e2423.

Howell, J. C., Watts, K. D., Parker, M. W., Wu, J., Kollhoff, A., Wingo, T. S., et al. (2017). Race modifies the relationship between cognition and Alzheimer's disease cerebrospinal fluid biomarkers. Alzheimers Res. Ther. 9:88.

Khalil, M., Teunissen, C. E., Otto, M., Piehl, F., Sormani, M. P., Gattringer, T., et al. (2018). Neurofilaments as biomarkers in neurological disorders. Nat. Rev. Neurol. 14, 577-589.

Lees, R., Selvarajah, J., Fenton, C., Pendlebury, S. T., Langhorne, P., Stott, D. J., et al. (2014). Test accuracy of cognitive screening tests for diagnosis of dementia and multidomain cognitive impairment in stroke. Stroke 45, 3008-3018. doi: 10.1161/strokeaha.114.005842

Liu, D., Chen, J., Wang, X., Xin, J., Cao, R., and Liu, Z. (2020). Serum neurofilament light chain as a predictive biomarker for ischemic stroke outcome: a systematic review and meta-analysis. J. Stroke Cerebrovasc. Dis. 29:104813. doi: 10.1016/j. jstrokecerebrovasdis.2020.104813

Lycke, J. N., Karlsson, J. E., Andersen, O., and Rosengren, L. E. (1998). Neurofilament protein in cerebrospinal fluid: a potential marker of activity in multiple sclerosis. J. Neurol. Neurosurg. Psychiatry 64, 402-404. doi: 10.1136/ jnnp.64.3.402

Mattsson, N., Andreasson, U., Zetterberg, H., and Blennow, K. (2017). Association of plasma neurofilament light with neurodegeneration in patients with alzheimer disease. JAMA Neurol. 74, 557-566. doi: 10.1001/jamaneurol.2016. 6117

Nielsen, H. H., Soares, C. B., Høgedal, S. S., Madsen, J. S., Hansen, R. B., and Christensen, A. A. (2020). Acute neurofilament light chain plasma levels correlate with stroke severity and clinical outcome in ischemic stroke patients. Front. Neurol. 11:448. doi: 10.3389/fneur.2020.00448

Olsson, B., Portelius, E., Cullen, N. C., Sandelius, Å, Zetterberg, H., Andreasson, U., et al. (2019). Association of cerebrospinal fluid neurofilament light protein levels with cognition in patients with dementia, motor neuron disease, and movement disorders. JAMA Neurol. 76, 318-325. doi: 10.1001/jamaneurol. 2018.3746

Onatsu, J., Vanninen, R., Jäkälä, P., Mustonen, P., Pulkki, K., Korhonen, M., et al. (2019). Serum neurofilament light chain concentration correlates with infarct volume but not prognosis in acute ischemic stroke. J. Stroke Cerebrovasc. Dis. 28, 2242-2249. doi: 10.1016/j.jstrokecerebrovasdis.2019.05.008

Palermo, G., Mazzucchi, S., Della Vecchia, A., Siciliano, G., Bonuccelli, U., Azuar, C., et al. (2020). Different clinical contexts of use of blood neurofilament light chain protein in the spectrum of Neurodegenerative Diseases. Mol. Neurobiol. 57, 4667-4691. doi: 10.1007/s12035-020-02035-9

Pedersen, A., Stanne, T. M., Nilsson, S., Klasson, S., Rosengren, L., Holmegaard, L., et al. (2019). Circulating neurofilament light in ischemic stroke: temporal profile and outcome prediction. J. Neurol. 266, 2796-2806. doi: 10.1007/s00415-01909477-9
Pendlebury, S. T., Rothwell, P. M., and Oxford Vascular, S. (2019). Incidence and prevalence of dementia associated with transient ischaemic attack and stroke: analysis of the population-based Oxford Vascular Study. Lancet Neurol. 18, 248-258. doi: 10.1016/s1474-4422(18)30442-3

Quiroz, Y. T., Zetterberg, H., Reiman, E. M., Chen, Y., Su, Y., Fox-Fuller, J. T., et al. (2020). Plasma neurofilament light chain in the presenilin 1 E280A autosomal dominant Alzheimer's disease kindred: a cross-sectional and longitudinal cohort study. Lancet Neurol. 19, 513-521. doi: 10.1016/s1474-4422(20)30137-x

Rohrer, J. D., Woollacott, I. O., Dick, K. M., Brotherhood, E., Gordon, E., Fellows, A., et al. (2016). Serum neurofilament light chain protein is a measure of disease intensity in frontotemporal dementia. Neurology 87, 1329-1336. doi: 10.1212/wnl.0000000000003154

Rosengren, L. E., Karlsson, J. E., Sjögren, M., Blennow, K., and Wallin, A. (1999). Neurofilament protein levels in CSF are increased in dementia. Neurology 52 , 1090-1093. doi: 10.1212/wnl.52.5.1090

Sjögren, M., Blomberg, M., Jonsson, M., Wahlund, L. O., Edman, A., Lind, K., et al. (2001). Neurofilament protein in cerebrospinal fluid: a marker of white matter changes. J. Neurosci. Res. 66, 510-516. doi: 10.1002/jnr.1242

Skillbäck, T., Farahmand, B., Bartlett, J. W., Rosén, C., Mattsson, N., Nägga, K., et al. (2014). CSF neurofilament light differs in neurodegenerative diseases and predicts severity and survival. Neurology 83, 1945-1953. doi: 10.1212/wnl. 0000000000001015

Tiedt, S., Duering, M., Barro, C., Kaya, A. G., Boeck, J., Bode, F. J., et al. (2018). Serum neurofilament light: a biomarker of neuroaxonal injury after ischemic stroke. Neurology 91, e1338-e1347.

Traenka, C., Disanto, G., Seiffge, D. J., Gensicke, H., Hert, L., Grond-Ginsbach, C., et al. (2015). Serum neurofilament light chain levels are associated with clinical characteristics and outcome in patients with cervical artery dissection. Cerebrovasc. Dis. 40, 222-227. doi: 10.1159/000440774

Uphaus, T., Bittner, S., Gröschel, S., Steffen, F., Muthuraman, M., Wasser, K., et al. (2019). NfL (Neurofilament Light Chain) levels as a predictive marker for long-term outcome after ischemic stroke. Stroke 50, 3077-3084.

Wallin, A., and Sjögren, M. (2001). Cerebrospinal fluid cytoskeleton proteins in patients with subcortical white-matter dementia. Mech. Ageing Dev. 122, 1937-1949. doi: 10.1016/s0047-6374(01)00306-2

Weston, P. S. J., Poole, T., Ryan, N. S., Nair, A., Liang, Y., Macpherson, K., et al. (2017). Serum neurofilament light in familial Alzheimer disease: a marker of early neurodegeneration. Neurology 89, 2167-2175. doi: 10.1212/ wnl.0000000000004667

Zetterberg, H. (2016). Neurofilament light: a dynamic cross-disease fluid biomarker for neurodegeneration. Neuron 91, 1-3. doi: 10.1016/j.neuron.2016. 06.030

Zetterberg, H., Hietala, M. A., Jonsson, M., Andreasen, N., Styrud, E., Karlsson, I., et al. (2006). Neurochemical aftermath of amateur boxing. Arch. Neurol. 63, 1277-1280. doi: 10.1001/archneur.63.9.1277

Zetterberg, H., Skillbäck, T., Mattsson, N., Trojanowski, J. Q., Portelius, E., Shaw, L. M., et al. (2016). Association of cerebrospinal fluid neurofilament light concentration With Alzheimer Disease progression. JAMA Neurol. 73, 60-67. doi: 10.1001/jamaneurol.2015.3037

Zhang, X., and Bi, X. (2020). Post-stroke cognitive impairment: a review focusing on molecular biomarkers. J. Mol. Neurosci. 70, 1244-1254. doi: 10.1007/ s12031-020-01533-8

Zhao, Y., Xin, Y., Meng, S., He, Z., and Hu, W. (2019). Neurofilament light chain protein in neurodegenerative dementia: a systematic review and network metaanalysis. Neurosci. Biobehav. Rev. 102, 123-138. doi: 10.1016/j.neubiorev.2019. 04.014

Conflict of Interest: The authors declare that the research was conducted in the absence of any commercial or financial relationships that could be construed as a potential conflict of interest.

Copyright $\odot 2021$ Wang, Wang, Li, Li, Zhang, Jiang, Fan, Wang and Yang. This is an open-access article distributed under the terms of the Creative Commons Attribution License (CC BY). The use, distribution or reproduction in other forums is permitted, provided the original author(s) and the copyright owner(s) are credited and that the original publication in this journal is cited, in accordance with accepted academic practice. No use, distribution or reproduction is permitted which does not comply with these terms. 